\title{
Methylprednisolone as adjuvant in treatment of acute respiratory distress syndrome owing to leptospiro- sis-a pilot study
}

\author{
Abraham Ittyachen, V. K. Lakshmanakumar, C. K. Eapen, M. R. Joseph
}

\begin{abstract}
Background: Acute respiratory distress syndrome (ARDS) owing to leptospirosis is associated with a high mortality. This is in spite of mechanical ventilation in a critical care setting. ARDS by its very nature, associated with economic constraints in our patients has made treating these patients a challenge. Objective: To study if combination of methylprednisolone and noninvasive ventilation is life-saving in ARDS owing to leptospirosis. Design: Interventional pilot study. Setting: Medical intensive care unit of a rural medical college hospital. Participants: Eight patients diagnosed to have ARDS owing to leptospirosis. Main outcome indicator: Survival. Results: Seven out of eight patients who were treated with methylprednisolone and noninvasive ventilation recovered fully to resume normal life. Conclusion: Methylprednisolone with noninvasive ventilation is life-saving in ARDS owing to leptospirosis.
\end{abstract}

Key Words: ARDS, Leptospirosis, Methylprednisolone, Noninvasive ventilation

\section{Introduction}

Pulmonary involvement in leptospirosis varies in its incidence and presentation, incidence varying from as low as $20 \%$ to as high as $70 \%$. It may be mild in the form of a cough or take extreme forms as alveolar hemorrhage and acute respiratory distress syndrome (ARDS). ${ }^{[1]}$ The extreme forms have always been associated with a high mortality, in spite of invasive mechanical ventilation in a critical-care setting. ${ }^{[2,3]}$

Our hospital is situated in a rural area endemic for leptospirosis. In an earlier series the major complications of leptospirosis in our patients have been reported as re-

\section{From:}

Division of Critical Care \& Emergency Services, Department of Medicine,

M. O. S. C. Medical College, Kolenchery, Kerala State, India

Correspondence:

Dr. Abraham Ittyachen, Division of Critical Care \& Emergency Services,

M. O. S. C. Medical College, Kolenchery, Kerala State, India.

E-mail: abyliz@rediffmail.com nal failure $(16 \%)$, cardiac failure $(3 \%)$, coma or seizures $(3 \%)$, respiratory failure $(2 \%)$, and thrombocytopenia $(10 \%) .{ }^{[4]}$ We have observed that ARDS as a cause of respiratory failure leads to $100 \%$ mortality. ${ }^{[4]}$ Most of our patients come from the lower strata of society having a per capita monthly income of less than Rs.2500 and often lack insurance cover. ARDS by its very nature coupled with economic constraints has made treating these patients a challenge. Noninvasive ventilation has been used as an alternative to invasive ventilation in ARDS. ${ }^{[5]}$ In Jan 2004, we introduced noninvasive ventilation as an affordable alternative to treat ARDS but the mortality rate remained unchanged. It was in this background that we decided to use a combination of methylprednisolone and noninvasive ventilation to treat ARDS owing to leptospirosis. Though definite data is lacking, steroids especially methylprednisolone has also been used to treat ARDS. ${ }^{[6]}$ The sanction of the hospital ethics committee was obtained before initiation of the study. 


\section{Materials and Methods}

The study was done between June 2004 and November 2004-this is the monsoon season in Kerala and the incidence of leptospirosis peaks around this time of the year.

\section{Sample size}

During the period of the study there were eight patients with ARDS owing to leptospirosis. All of them had ARDS at the time of presentation. The diagnosis of leptospirosis was based on WHO guidelines ${ }^{[7]}$ and the diagnosis of ARDS was based on the North American-European Consensus Conference criteria..$^{[8]}$ The patients were treated in the medical intensive care unit (ICU).

\section{Treatment schedule}

All patients received injections of 1.5 million units IV Q6H crystalline penicillin and $500 \mathrm{mg}$ IV Q24H levofloxacin for 7 days. Artificial ventilatory support was simultaneously provided with a noninvasive ventilator (Respironics) - inspiratory positive airway pressure of 14 $\mathrm{cm} \mathrm{H}_{2} \mathrm{O}$ and expiratory positive airway pressure of $6 \mathrm{~cm}$ $\mathrm{H}_{2} \mathrm{O}$. In addition, all patients in the treatment group were initially started on injection of methylprednisolone (40 $\mathrm{mg}$ ) IV Q6H. The vital parameters of the patient were monitored in theICU by the resident. Initially, oxygen was given at a high rate of $30 \mathrm{l} / \mathrm{min}$. Oxygen flow was titrated to maintain an oxygen saturation of more than $95 \%$. When the respiratory rate fell below $25 / \mathrm{min}$ and the oxygen requirement fell below $10 \mathrm{l} / \mathrm{min}$, the noninvasive ventilator was disconnected and the patient was connected to a high-concentration oxygen mask with reservoir (nonrebreathing mask). At this point the dosing interval of methylprednisolone was made every $8 \mathrm{~h}$. Subsequently, depending on the clinical response (oxygen saturation and respiratory rate) oxygen delivered and methylprednisolone were titrated down (see treatment algorithm) (Figure 1).

Chest radiographs were taken on the day of admission and repeated on the second day. Further chest Xrays were taken only after the patient was taken off the noninvasive ventilator. For those who had thrombocytopenia (defined as platelet count less than $100,000 / \mathrm{mm}^{3}$ ) and renal failure (defined as $\mathrm{S}$. Creatinine of more than $1.4 \mathrm{mg} \%)$, platelet count and S. creatinine were repeated every day.

\section{Outcome indicator}

Survival was taken as the outcome indicator

\section{Results}

Three of our patients were males and five were females (Table 1). The youngest patient was 15 years old and the oldest was 53 years old. Six out of seven patients required an ICU stay of less than 6 days; only one required more than 6 days. As for the total hospital stay, only one patient required a hospital stay of more than 2 weeks.

One of our patients developed respiratory failure and was advised invasive ventilation but refused to receive the same and noninvasive ventilation was continued; this patient expired while on the noninvasive ventilator. All the other patients recovered fully and were back to normal routine in less than 2 weeks (Table 2 ).

We observed that in all patients except one there was an increase in the infiltrates on the chest X-ray when taken on the second day after the initiation of crystalline penicillin. Seven of our patients had renal failure; all of them were nonoliguric. None of them needed dialysis and all of them recovered. Seven patients also had thrombocytopenia; none of them had any bleeding complications. All of them recovered during the course of treatment. None of the patients had any complication regarding noninvasive ventilation or administration of methylprednisolone.

Majority of our patients (five out of seven) required methylprednisolone for 4 days. For most it took another 1-2 days more for full resolution of the chest X-ray. Use of the noninvasive ventilator was usually 1 day less than the duration of methylprednisolone administration (Figure 2).

\section{Table 1: Patient characteristics}

\begin{tabular}{lll}
\hline Age and sex distribution & No. \\
Age & $<45$ & 6 \\
& $>45$ & 2 \\
Sex & Male & 3 \\
& Female & 5 \\
ARDS at presentation & 8 \\
\hline
\end{tabular}

\begin{tabular}{lllc}
\hline \multicolumn{4}{l}{ Table 2: } \\
\hline Outcome of treatment \\
\hline Outcome & Survived & Died & Total \\
No. & $7(87.5 \%)$ & $1^{\mathrm{a}}(12.5 \%)$ & 8 \\
\hline
\end{tabular}

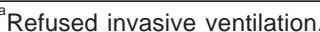




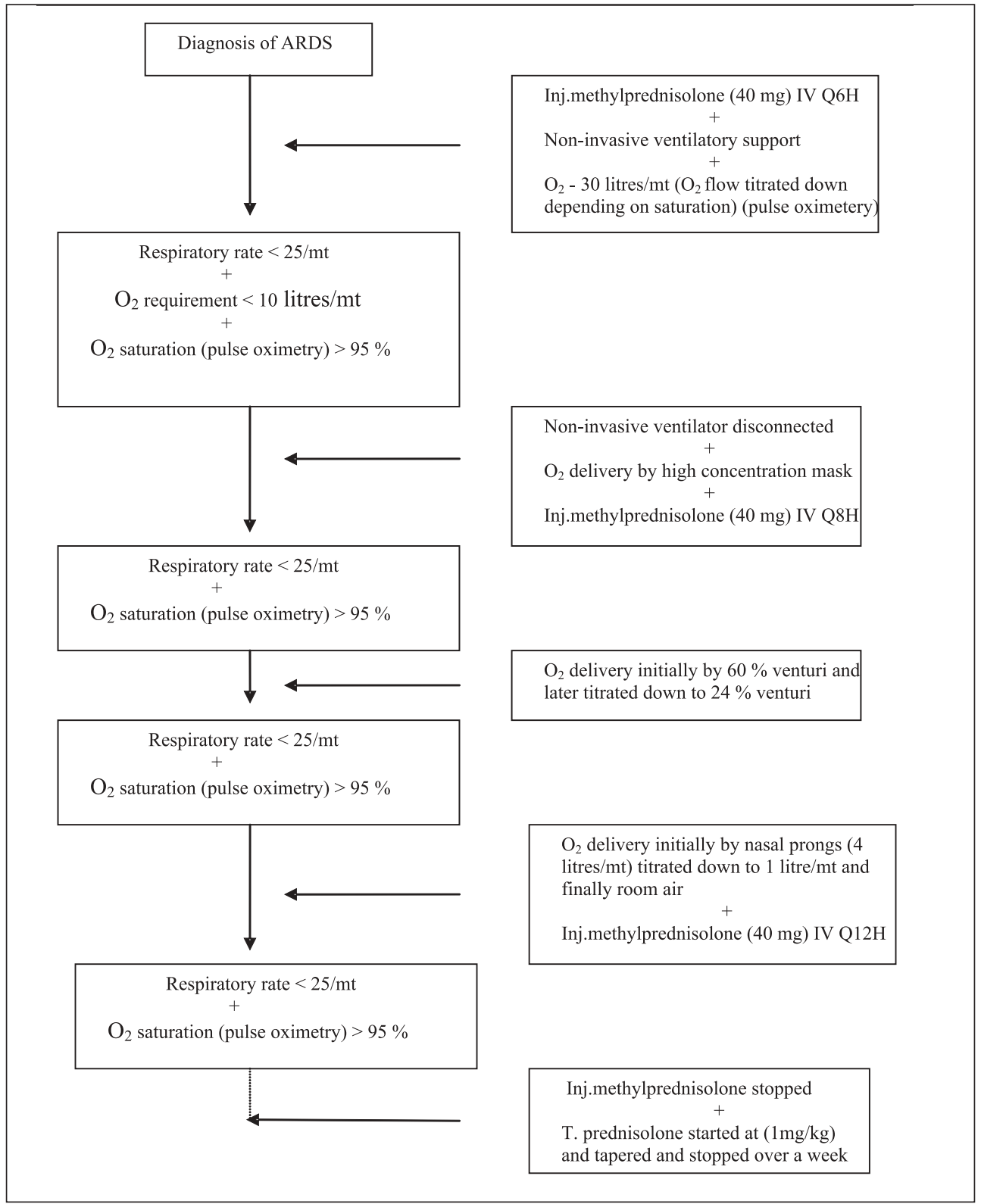

Figure 1: Treatment algorithm

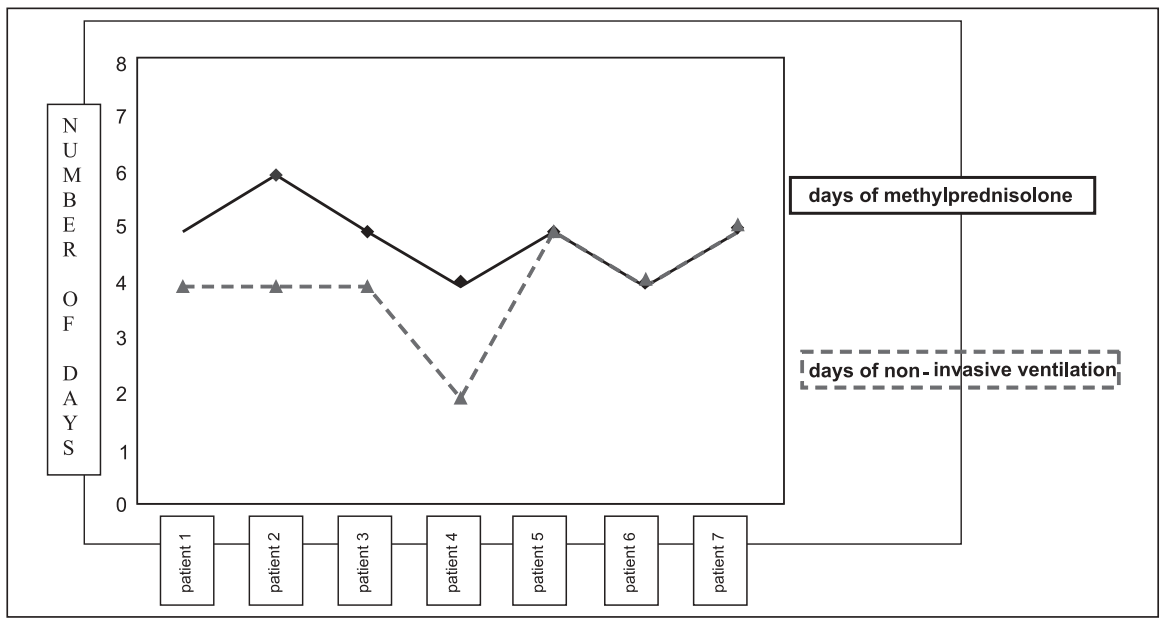

Figure 2: Graph showing number of days of treatment for all patients who survived 


\section{Discussion}

The involvement of the lung in leptospirosis is well known, and one of the most commonly held views regarding this are the involvement of mediators. ${ }^{[9-12]}$ There is also some evidence that this may be a direct effect of the organism on the lung tissue. ${ }^{[13]}$ The increase in infiltrates which we observed on the chest $\mathrm{X}$-ray when taken on the second day after the initiation of penicillin is probably mediator-related; we hypothesize this to be a Jarisch-Herxheimer-type reaction. As with other spirochetal infections Jarisch-Herxheimer reaction is also described in leptospirosis. ${ }^{[14]}$ Owing to the involvement of mediators and associated inflammatory process there is a potential role for steroids. Steroids have been used in the late phase of ARDS; ;6,15] however, its use in the early phase has been unproven. In this study we have found that high-dose steroids in combination with noninvasive ventilator is beneficial in ARDS owing to leptospirosis. Economical and infrastructural constraints in a rural hospital like ours often limit the use of treatment modalities such as invasive ventilation. Even in those who were treated with invasive ventilation mortality was $100 \%$ in our earlier experience. ${ }^{[4]}$ On the other hand, this study shows that ARDS owing to leptospirosis can be treated successfully with less expensive and simpler interventions.

\section{Conclusion}

Methylprednisolone in ARDS (owing to leptospirosis) in combination with respiratory support by noninvasive ventilation is life-saving.

In our population (and elsewhere), for those at risk for leptospirosis, the above combination therapy offers an affordable and successful therapeutic measure without the "cost and complications" that would otherwise accompany invasive ventilation.

\section{References}

1. Paul NL. Leptospirosis. Clin Microbiol Rev 2001;14:296-326.

2. Marotto PC, Nascimento CM, Eluf-Neto J, Marotto MS, Andrade L, Sztajnbok J, et al. Acute lung injury in leptospirosis: clinical and laboratory features, outcome, and factors associated with mortality. Clin Infect Dis 1999;29:1561-3.

3. Carvalho CR, Bethlem EP. Pulmonary complications of leptospirosis. Clin Chest Med 2002; 23:469-78.

4. Kuriakose M, Eapen CK, Paul R. Leptospirosis in Kolenchery, Kerala, India: epidemiology, prevalent local serogroups and serovars and a new serovar. Eur J Epidemiol 1997;13:691-7.

5. Rocker GM, Mackenzie MG, Williams B, Logan PM. Noninvasive positive pressure ventilation: successful outcome in patients with acute lung injury/ARDS. Chest 1999;115:173-7.

6. Kallet R.H. Evidence-based management of acute lung injury and acute respiratory distress syndrome. Respir Care 2004;49:793-809.

7. Guidelines for the Control of Leptospirosis, Geneva, World Health Organisation, 1982, WHO Offset Publication No. 67.

8. Bernard GR, Artigas A, Brigham KL, Carlet J, Falke K, Hudson $\mathrm{L}$, et al. The American-European Consensus Conference on ARDS. Definitions, mechanisms, relevant outcomes, and clinical trial coordination. Am J Respir Crit Care Med 1994;149:81824.

9. Bethlem EP, Carvalho CR. Pulmonary leptospirosis. Curr Opin Pulm Med 2000;6:436-41.

10. Marotto PC, Nascimento CM, Eluf-Neto J, Marotto MS, Andrade L, Sztajnbok J, et al. Acute lung injury in leptospirosis: clinical and laboratory features, outcome, and factors associated with mortality. Clin Infect Dis 1999;29:1561-3.

11. Carvalho CR, Bethlem EP. Pulmonary complications of leptospirosis. Clin Chest Med 2002;23:469-78.

12. Martinez Garcia MA, de Diego Damia A, Menendez Villanueva R, Lopez Hontagas JL. Pulmonary involvement in leptospirosis. Eur J Clin Microbiol Infect Dis 2000;19:471-4.

13. Silva JJ, Dalston MO, Carvalho JE, Setubal S, Oliveira JM, Pereira MM. Clinicopathological and immunohistochemical features of the severe pulmonary form of leptospirosis. Rev Soc Bras Med Trop 2002;35:395-9.

14. Friedland JS, Warrell DA. The Jarisch-Herxheimer reaction in leptospirosis: possible pathogenesis and review. Rev Infect Dis 1991;13:207-10.

15. Umberto Meduri G, Stacey Headley A, Golden E, Carson SJ, Reba A, Umberger, et al. Effect of prolonged methylprednisolone therapy in unresolving acute respiratory distress syndrome. A randomized controlled trial. JAMA 1998;280:159-65. 